\title{
NUMERICAL INVESTIGATION OF OPTICAL BURST SWITCHING
}

The simulation analysis of a physical layer of the "all optical burst switching" using a VPI Photonics ${ }^{T M}$ simulation program is presented in this article. The optical burst networks adopt dedicated control channel to transmit headers of burst, at the same time the payload is transmitted on the other optical data channels after the offset time. We have proposed OBS network with four data channels and one control channel consisting of the following main optical elements: lasers with external modulation, a wavelength converter using Kerr effect cross phase modulation (XPM) in optical semiconductor amplifiers (SOA ), a wavelength switch $4 \times 4$ and photodetector. We present the main connection of the OBS network, data and control message flow, processing in the core node and sending to next node. The results are visualized in graphs.

\section{Introduction}

Despite of the fact, that the optical burst switching (OBS) concept has been known since $1980 \mathrm{~s}^{1)}$, it has not had a great success in electrical domain. The main reason for it was great complexity and requirements to run burst switching which are comparable with packet switching and the resulting flexibility is lower. However, deploying high capacity WDM links have led to new possibilities for utilisation of this principle and OBS is one of promising solutions for WDM networks [1, 2, 4].

The main characteristic of OBS is [5]:

- User data (IP packets) are accumulated in a constant or variable length of the optical burst, which would have up to one hundred $\mathrm{kB}$.

- Separation between the headers (control information) and the payloads is in space and time. The heads are sent to the other OBS nodes in a single wavelength channel and are in all OBS nodes processed in electrical domain. The headers set OBS node for receiving and routing inbound payload burst to the next node.

- The burst data are switched in the OBS nodes asynchronously whereby remain in optical domain while required target is reached.

- The sources are allocated with using the one-way reservation, i.e. the burst is not sent while confirmation about successful set end to end way is not received

\section{The simulation scheme of the OBS node}

The simulation scheme in VPI Photonics TM is shown in Fig. 1. Four data wavelengths and one control channel use external modulated lasers with on/off modulation. The optical channels are setup on $1 \mathrm{~mW}$ power and the type of code is non return to zero. The channels' wavelengths were set with respect to the ITU recommendation for DWDM channels in C band with $0.8 \mathrm{~nm}(100 \mathrm{GHz})$ separation. Four wavelengths $(1555.2 \mathrm{~nm}, 1554.4 \mathrm{~nm}, 1553.6 \mathrm{~nm}$, and $1555.8 \mathrm{~nm}$ ) were used for data channels and wavelength 1556 $\mathrm{nm}$ was set up for control channel. After multiplexing in DWDM multiplexer (insertion loss $2 \mathrm{~dB}$ ), data were sent into a single mode fibre with $50 \mathrm{~km}$ length, with attenuation of $0.2 \mathrm{~dB}^{-1} \mathrm{~km}^{-1}$ and dispersion of $16.6 \mathrm{ps} . \mathrm{km}^{-1} \mathrm{~nm}^{-1}$. The dispersion compensation was realised by the dispersion compensation fibre with the length of $10 \mathrm{~km}$ and strong negative dispersion. Signals then pass through the optical amplifier with $12 \mathrm{~dB}$ amplification of an optical signal. Followed by the optical signal demultiplexing (power loss $2 \mathrm{~dB}$ ) in the data and control channels, each channel was filtered by a bandpass filter with a Gaussian transfer function. The control signal was detected with the PIN photodetector. After the reconstruction of signal timing and elimination of d.c. component the signal went through a level comparator and at the control input of $4 \times 4$ wavelength switch (Fig. 2). According to the bit combination at the control input data burst was switched to the specific output $[3,6$, 7]

Whereas the control signal was processed electronically, the optical signals are after sideband filtration brought into a wavelength converter. The wavelength converter, which uses nonlinear Kerr effects as XPM in SOA, was simulated in Mach-Zehnder interferometric structure. The advantage of this scheme is in utilisation of XPM as a main effect. In a such case not as high power of optical signals is needed as in the case of XGM. Brillouin and Raman scattering was avoided. Due to the other aspects, the amplification was needed to keep high SNR [6].

\footnotetext{
* Milan Dado, Stanisalv Krajci, Jozef Dubovan ${ }^{1}$, Dusan Sabol ${ }^{2}$

${ }^{1}$ Department of Telecommunications, Faculty of Electrical Engineering, University of Zilina, Slovakia, Milan.Dado@fel.uniza.sk,

${ }^{2}$ University College Dublin, Dept. of Electrical, Electronical and Mechanical Engineering, Ireland, dsabol@centrum.sk

1) OBS was designed after 1990s and it was focused on new reservation protocol for accumulating data in bursts, prototypes and architectures [5].
} 


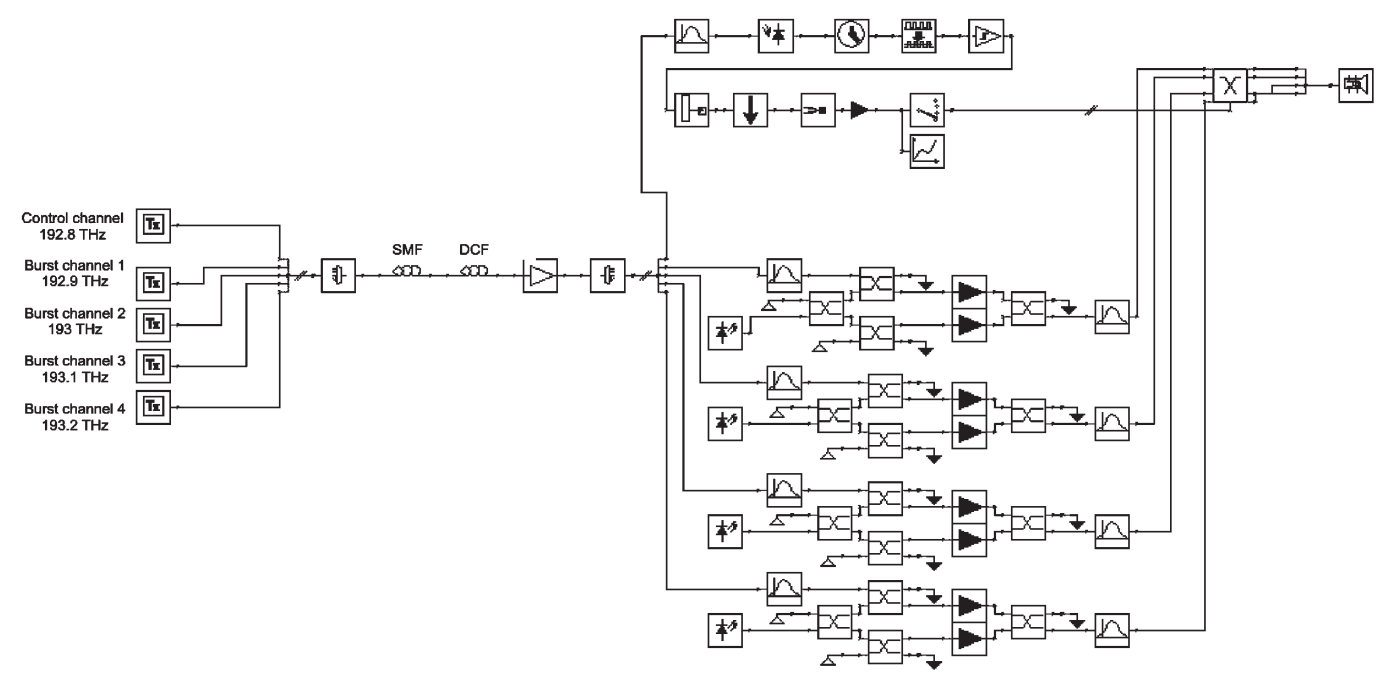

Fig. 1 Simulation scheme of OBS node

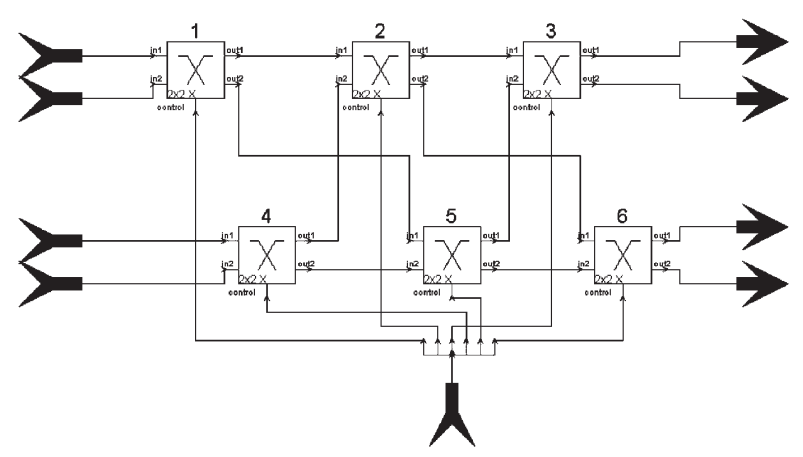

Fig. 2 Wavelength switch $4 x 4$

\section{Simulation}

The OBS node sends data burst in time offset in accordance with the header. The delay of the time offset is possible to perform directly by transmission either from the edge nodes of network or from FDL lines in backbone nodes. In our case the delay is performed from the edge nodes in network $[5,7,9]$.

In Fig. 3 there is a combination of 6 bits and 4 control headers, which were created in transmitters of control messages for each data channel. Table 1 shows relevant switch-over in dependence on these bits.

Switching in dependence on combination

Table 1 of bits in the burst heads

\begin{tabular}{|c|c|c|c|c|c|}
\hline & Burst head & Input 1 in & Input 2 in & Input 3 in & Input 4 in \\
\hline 1. & 111001 & 1 & & & \\
\hline 2. & 110011 & & 4 & & \\
\hline 3. & 111010 & & & 2 & \\
\hline 4. & 101110 & & & & 3 \\
\hline
\end{tabular}

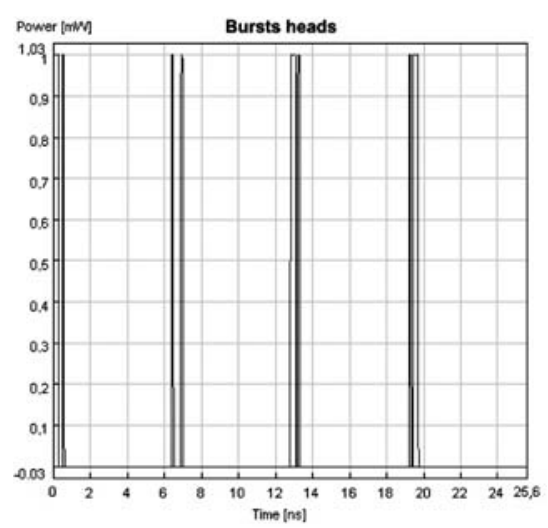

Fig. 3 Burst heads for four data channels

In case of simulation $10 \mathrm{Gbit.s}^{-1}$ bit rate the time length of one bit is $0.1 \mathrm{~ns}$. This means that all the bursts are delayed behind

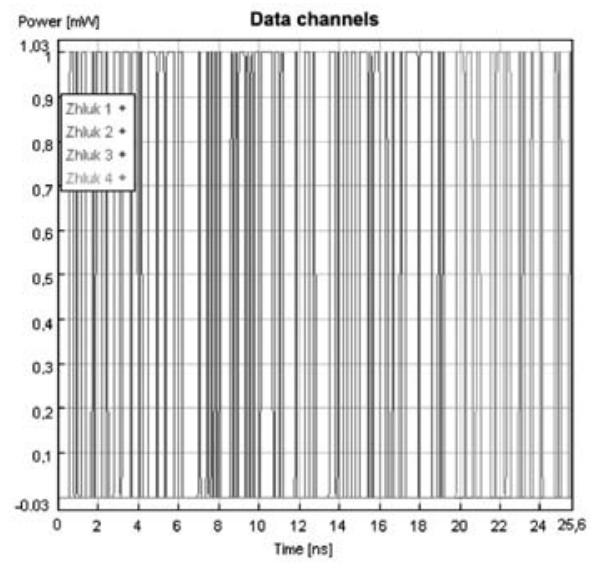

Fig. 4 Bursts with delay 0.6 ns after head 
the headers in sources by 0.6 ns (see Fig. 4). The bit length of a sequence is constant and was set up on 58 bits.

After overcoming the optical way with a length of $60 \mathrm{~km}$, the optical signal is amplified (12 dB). As can be seen in Fig. 5, SNR is sufficient for the next signal processing.

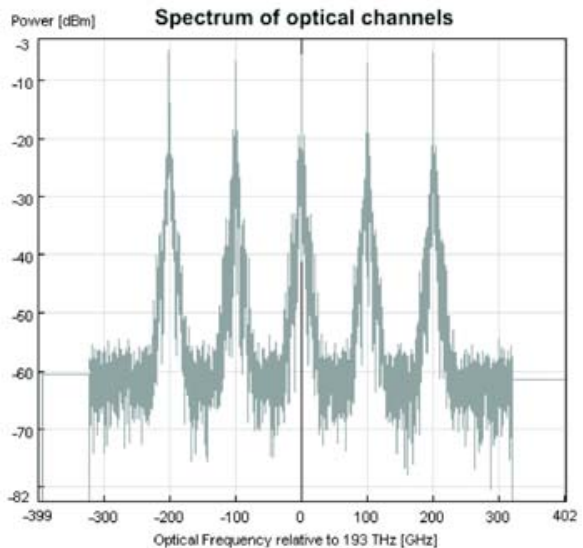

Fig. 5 Spectrum of optical channels after amplification

\section{Header processing}

After demultiplexing the channels, the control channel was linked to the optical band-pass filter and into the blocks for optoelectrical conversion and processing. A PIN photodiode is used as a photodetector. A very good eye diagram opening can be clearly seen in Fig. 6

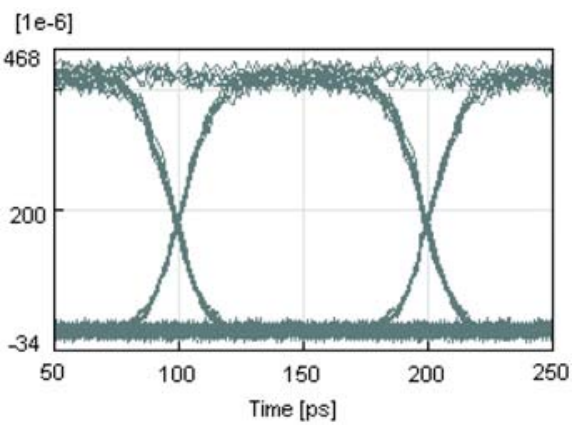

Fig. 6 Eye diagram of header after photodetector

The eye opening represents an influence (interference) between the channels. Crosstalk between the channels is low because an impulse spread is compensated by a dispersion fibre and the channel spacing of $100 \mathrm{GHz}$ was sufficient. In the lower and upper parts of the eye diagram a superposed noise can be seen, which is caused by characteristic attributes of the PIN diode and fibre nonlinearity. In Fig. 7 a received control message of first burst is depicted.

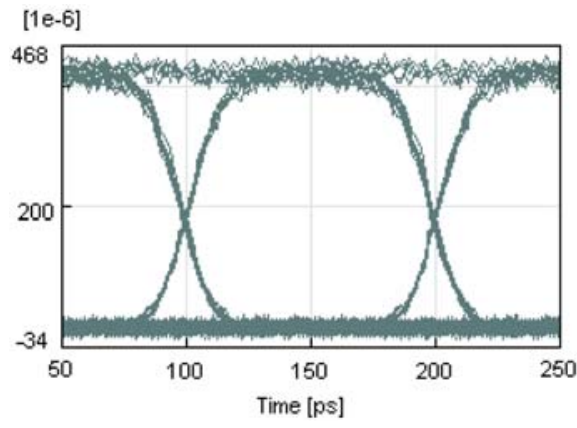

Fig. 7 Control message of first burst

\section{The wavelength conversion of the optical data chanels}

When the optical signal passes along optical fibre and demultiplexer and before switching to output, the signal goes through SOAs connected in Mach-Zehnder interferometric structure. An optical filter is connected for original wavelength filtering on each output of converter.

The best results for the conversion were achieved when an input power of the optical channel was set up on the half of power of the converted wavelength. The final values for SOA settings are in table 2 .

Settings for the SOA

Table 2

\begin{tabular}{|c|c|}
\hline \multicolumn{2}{|c|}{ OA values } \\
\hline Length $[\mu \mathrm{m}]$ & 500 \\
\hline Heigth $[\mu \mathrm{m}]$ & 3 \\
\hline Width $[\mathrm{nm}]$ & 80 \\
\hline Input power $[\mu \mathrm{W}]$ & 400 \\
\hline
\end{tabular}

The next effect which can be observed by conversion is an amplification by SOA of the input signal.

The input and output power was measured with a visualiser and a gain value of SOA was adjusted on $28 \mathrm{~dB}$. The time graph, eye diagram input and the converted channel can be seen in Figs. 8 and 9.

\section{A wavelength switch of the optical channels}

The last block of the designed OBS node is a wavelength switch $4 \times 4$ controlled by the control bits. One input to one output can be switched at the same time only. The MEMS was chosen from more potential switches for the OBS networks. An advantage of MEMS is a low insertion loss (about $0.2 \mathrm{~dB}$ ) and low crosstalk (less than $-60 \mathrm{~dB}$ ). After receiving electronical bits on the control input (see table 1) the inputs of an optical switch are switched to the desirable direction (Fig. 9). 

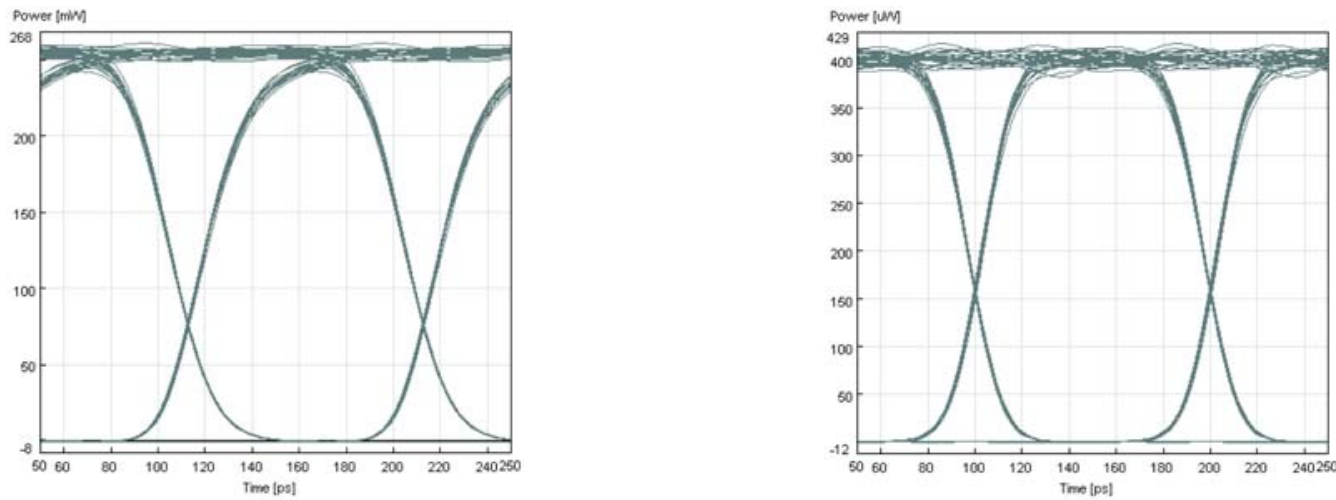

Fig. 8 Eye diagram before and after wavelength conversion

a)

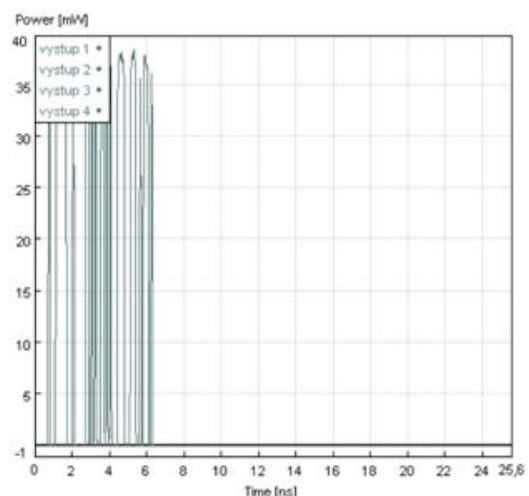

b)

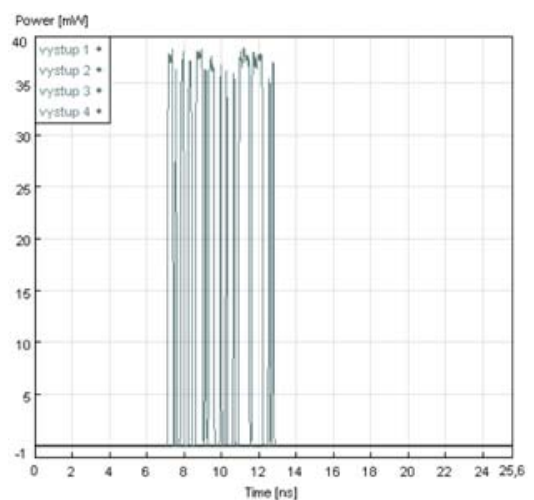

Fig. 9 Outputs of optical switch, a) 1. burst, b) 2. burst,

The channel spectral diagram at the output port of switch is shown in Fig. 10

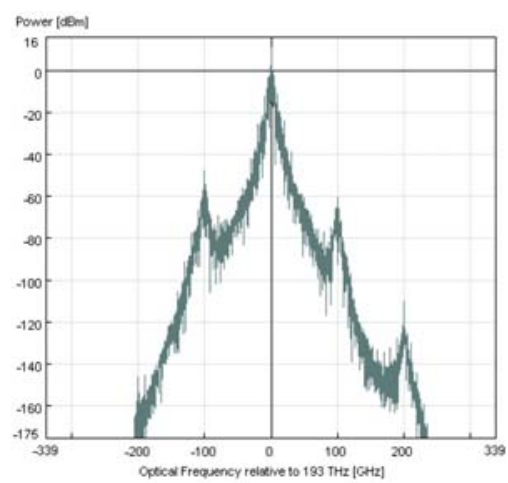

Fig. 10 Crosstalk from another channels

\section{Conclusion}

Optical burst networks are promising technology of the near future. The main advantage is a possibility of full transparent optical transmission between the communication nodes and the results are higher bit rates between these nodes. Another benefit of OBS usage is possibility to utilize an older infrastructure with upgrading existing WDM networks $[5,8]$.

The OBS node was created in a simulation environment (wavelength conversion with SOA, header processing, wavelength switch controlled by control signal, etc.) as mathematical models [7]. The main goal was to achieve results which would be usable for prediction of systems parameters in a real situation. Due to this fact all the parameters of each block used in the presented simulation model were set as parameters of real components.

The research presented in this paper was done with the support of COST 291 action, APVV project COST-0041-06 and APVV project "Technologies for optical signal processing for the next generation optical digital netwotks". 


\section{COMMNICOIIONS}

\section{References}

[1] MOUFTAH, H. T.; Ho, P.-H.: Optical Networks: Architecture and Survivability, Boston: Kluwer Academic, 2003, ISBN 1-4020-7196-5

[2] MAIER, M.: Metropolitan A rea WDM Networks: An AWG Based Approach, Boston: Kluwer Academic, 2004, ISBN 1-4020-7574-X

[3] Next Generation Optical Network Design and Modeling: IFIP TC6/WG6.10 Sixth working conference on Optical Network Design and Modelling (ONDM 2002), February 4-6, 2002 Torino, Italy, Norwell, Massachusetts: Kluwer Academic Publishers, 2003, ISBN 1-4020-7371-2

[4] RAMAMURTHY, B.: Design of optical WDM networks: LAN, MAN and WAN architectures, Boston, Dordrecht: Kluwer Academic Publishers, 2001, ISBN 0-7923-7281-6

[5] JUE, J. P.; VOKKARANE, V. M.: Optical Burst Switched Networks, New York: Springer Science+Bussines Media, Inc., 2005, ISBN 0-387-23756-9

[6] ANAN, M. T.; CHAUDHRY, G. M.; BENHADDOU, D.: Architecture and Performance of A Next-Generation Optical Burst Switch (OBS), BROADNETS 2006 Third International Conference on Broadband Communications, Networks, and Systems, October 1-5, 2006, San José, California, USA

[7] FISCHER DE TOLEDO, M. C.; ZUCCHI, W. L.: Simulation of an optical burst switch using fiber delay lines, 2005 SBMO/IEEE MTT-S International Conference, July 25 to 28, 2005, Brasil

[8] YUHUA CH.; TURNER, J. S.; Mo, P.-F.: Optimal Burst Scheduling in Optical Burst Switched Networks, Journal of Lightwave Technology, vol. 25, No. 8, August 2007

[9] ROSTAMI, A.; WOLISZ, A.: Modeling and Synthesis of Traffic in Optical Burst-Switched Networks, Journal of Lightwave Technology, vol. 25, No. 10, October 2007 